
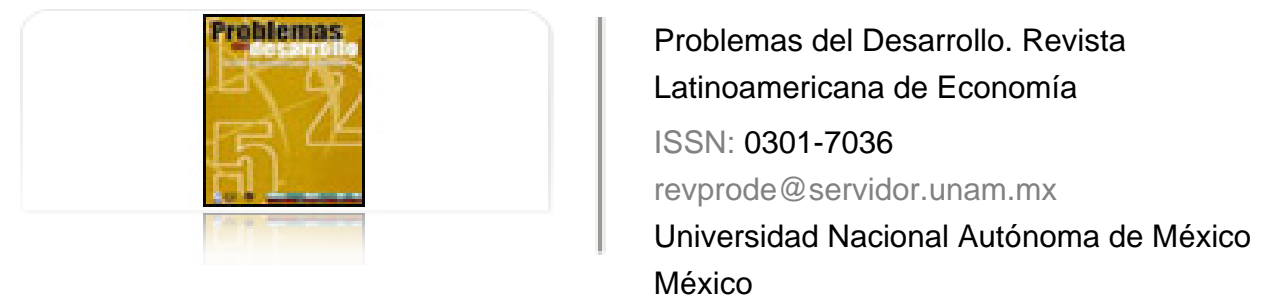

Velasco, Andrés Enrique Miguel; Maldonado Cruz, Pedro; Torres Valdez, Julio César

Desigualdad del desarrollo regional en México

Problemas del Desarrollo. Revista Latinoamericana de Economía, vol. 38, núm. 151, octubre-

diciembre, 2007, pp. 87-102

Universidad Nacional Autónoma de México

Distrito Federal, México

Disponible en: http://www.redalyc.org/articulo.oa?id=11820167005

- Cómo citar el artículo

Número completo

- Más información del artículo

Página de la revista en redalyc.org

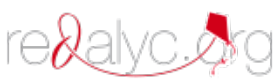

Sistema de Información Científica

Red de Revistas Científicas de América Latina, el Caribe, España y Portugal Proyecto académico sin fines de lucro, desarrollado bajo la iniciativa de acceso abierto 


\section{DESIGUALDAD DEL DESARROLLO REGIONAL EN MÉXICO}

\section{Andrés Enrique Miguel Velasco* Pedro Maldonado Cruz** Julio César Torres Valdez ${ }^{* * *}$}

Fecha de recepción: 7 de junio de 2007. Fecha de aceptación: 13 de agosto de 2007.

\section{Resumen}

El presente artículo analiza la manifestación de la equidad, la eficiencia y la sustentabilidad en las regiones de México, a partir de su desarrollo humano durante el periodo 1950-2003. Se propone como metodología la medición de las desigualdades regionales por medio de la entropía absoluta y relativa de los indicadores del desarrollo humano y la sustentabilidad. La hipótesis contrastada es que las desigualdades regionales producto del desarrollo han aumentado en México durante el periodo 1950-2003, y que en fechas recientes los desequilibrios identificados mediante la evaluación de la sustentabilidad regional aun tienden a ser mayores. Finalmente, trata de propiciar la reflexión acerca de cuáles pueden ser algunas de las causas que propician las desigualdades actuales de las grandes regiones mexicanas.

Palabras clave: región, desarrollo, desigualdades, desarrollo regional, desigualdades regionales.

* Profesor investigador del Instituto Tecnológico de Oaxaca. Correo electrónico: andmig56@ hotmail.com.

** Coordinador del doctorado en Desarrollo Regional y Tecnológico del Instituto Tecnológico de Oaxaca.Correo electrónico: pemece@itoaxaca.edu.mx y pemece@gmail.com.

*** Jefe de la División de Posgrado e Investigación en el Instituto Tecnológico de Oaxaca. Correos electrónicos: jcesartv@itoaxaca.edu.mx y jcesartv58@hotmail.com. 


\section{Abstract}

This article analyzes the manifestation of equity, efficiency and sustainability in Mexico's regions, starting from its human development during the period 1950-2003. As methodology it proposes the measurement of regional inequalities through the absolute and relative entropy of human development and sustainability indicators. The contrasted hypothesis is that the regional inequalities produced by development have increased in Mexico during the period 1950-2003, and that recently the disequilibria identified by the evaluation of regional sustainability tends to be greater still. Finally, it aims at promoting reflection on what might be some of the causes behind the present inequalities in Mexico's large regions.

Key words: region, development, inequalities, regional development, regional inequalities.

\section{Résumé}

Le présent article analyse les manifestations de la parité, de l'efficacité et de la durabilité du développement dans les régions du Mexique, à partir de leur développement humain durant la période 1950-2003. La méthodologie proposée consiste en la mesure des inégalités régionales au moyen de l'entropie absolue et relative des indicateurs de développement humain et de durabilité du développement. L'hypothèse contrastée est que les inégalités régionales de développement ont augmenté au Mexique durant la période considérée, et que récemment les déséquilibres perçus entre différentes régions sur le plan de la durabilité du développement tendent à s'accroître encore. Finalement, l'article tente de fomenter la réflexion quant aux causes possibles des inégalités actuelles entre les grandes régions du Mexique.

Mots clés: région, développement, inégalités, développement régional, inégalités entre régions.

\section{Resumo}

O presente artigo analisa a manifestação da equidade, a eficiência e a sustentabilidade nas regiões do México, a partir do seu desenvolvimento humano durante o período 1950-2003. Propõe-se como metodologia a medição das desigualdades regionais por meio da entropía absoluta e relativa dos indicadores de desenvolvimento humano e a sustentabilidade. A hipótese contrastada é que as desigualdades regionais produto do desenvolvimento aumentaram no México durante o período 1950-2003, e que em datas recentes os desequilíbrios identificados mediante a avaliação da sustentabilidade regional tendem a ser ainda maiores. Finalmente, trata-se de propiciar a reflexão sobre quais podem ser algumas das causas que propiciam as desigualdades atuais das grandes regiões mexicanas.

Palavras chave: região, desenvolvimento, desigualdades, desenvolvimento regional, desigualdades regionais. 


\title{
Introducción
}

\begin{abstract}
$\mathrm{n}$ el análisis del desarrollo regional todavía es un tema relevante el conocer C la manifestación de la equidad, la eficiencia y la sustentabilidad, pues estos tópicos dan cuenta del nivel de igualdad en el bienestar social, el crecimiento económico y el equilibrio con la naturaleza que proporciona el proceso de desarrollo. En este trabajo se analizan las desigualdades regionales ocasionadas por sus desequilibrios, al tomar como referencia el desarrollo de las regiones de México durante el periodo 1950-2003, concretamente por medio de la medición de la entropía del Índice de Desarrollo Humano del país. La hipótesis propuesta supone que las desigualdades regionales producto del desarrollo han aumentado en México durante dicho lapso. Se propone como metodología la medición de la entropía absoluta y relativa de los indicadores del desarrollo y la sustentabilidad para evaluar las desigualdades de las regiones del país.
\end{abstract} D

\section{Fundamentos conceptuales}

\section{Antecedentes: entropía y desarrollo regional}

En general, el desarrollo regional puede entenderse como "el proceso de cambio sostenido, que tiene como finalidad el progreso permanente de la región, de la comunidad regional como un todo y de cada individuo residente en ella" (Boisier, 1996). Existen diversos indicadores propuestos para medirlo, uno de ellos es el Índice de Desarrollo Humano, conceptualizado como la libertad de que gozan los individuos para elegir entre distintas opciones y formas de vida, mediante factores que permiten a la personas ser libres, como son la posibilidad de alcanzar una vida larga y saludable, el adquirir conocimientos individual y socialmente valiosos, y el tener la oportunidad de obtener los recursos necesarios para disfrutar de un nivel de vida decoroso (PNUD, 2004). Este índice posee la cualidad de su aceptación y estandarización en los ámbitos nacional e internacional, lo cual permite efectuar comparaciones en tiempo y espacio con el mismo. El desarrollo regional es el proceso generador de riqueza económica, de bienestar social, así como de sustentabilidad, y cuando se manifiesta en igualdad de oportunidades para todos — personas, sectores y regiones- tiende a reflejarse en la armonía de las propias ciudades y regiones. Cuando el desarrollo no es armónico, sacrifica a cualesquiera de estos componentes, pero sobre 
todo el bienestar y la sustentabilidad en aras del crecimiento económico. En este tipo de desarrollo se manifiestan de manera especial las desigualdades regionales, la falta de eficiencia productiva y la pérdida de competitividad, lo que se resume en la entropía que posee la región.

La noción de "entropía" originalmente remite a la termodinámica y a la mecánica estadística como el conjunto de fenómenos físicos que se derivan de la llamada segunda Ley de la Termodinámica, pero tras el éxito conocido por la Teoría Matemática de la Comunicación de Shannon (1948) — cuyos instrumentos de cálculo arrancan de las operaciones formales empleadas en la mecánica estadística—, la similitud operativa para medir los intercambios energéticos y las transmisiones de mensajes confluyó a unificar en un mismo modelo el estudio del cambio en la naturaleza, en la sociedad y, por extensión, en las regiones. En las alusiones que a ella se hace en las ciencias sociales, se quiere expresar la tendencia de cualquier organización a deteriorarse, por la gradual decadencia que de forma natural opera entre las fuerzas que la cohesionan.

Para entender las desigualdades regionales, en este país se han desarrollado numerosos trabajos en el ámbito regional sobre la hipótesis de convergencia, lo que ha generado un debate donde destaca la existencia de divergencias, sobre todo a partir de 1980. Entre los primeros estudios realizados para comprender el crecimiento económico regional resalta el trabajo de Appendinni y Murayama (1972), quienes explican el atraso regional como un producto histórico del proceso de desarrollo económico del país y de sus factores inerciales. Para el periodo 1970-1979 varios estudios mencionan la mejoría de los niveles de bienestar y para 1980-1989 se presenta un aumento de las disparidades asociadas a los choques externos y al viraje en el modelo de sustitución de importaciones hacia las exportaciones que se mantiene de 1990 a la fecha (véase Hernández Laos, 1984; Garza, 1997; Ruiz Chiapetto, 1997, y Ramírez, 1986). Aunque existen otros métodos para medir las desigualdades regionales, por ejemplo, en función de la desviación estándar o el método de cluster para comparar los datos estadísticos. Antecedentes de la aplicación de la entropía como medida de la desigualdad los encontramos en el "Estadístico T de Theil" (1967), quien utilizó la teoría de Shannon para construir una medida de igualdad sobre la renta individual, cuyas mediciones son aplicables al ámbito territorial. En el presente artículo se utiliza como medida de las desigualdades regionales la entropía generada por el proceso de desarrollo de las propias regiones, concebido éste como un proceso que abarca aspectos económicos, sociales, de bienestar y de sustentabilidad. Puede señalarse que el análisis propuesto en este 
trabajo es aplicable a la valoración de las desigualdades inter e intrarregionales, y es básicamente de carácter descriptivo.

\section{La equidad, la eficiencia, la sustentabilidad y la entropía}

Tradicionalmente se entiende por eficiencia la preferencia por los resultados econó-

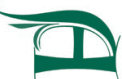
micos reflejados en el crecimiento económico, la mayor posición de los mercados por parte de las empresas, el incremento de la productividad, la reducción de los costos y el aumento de los beneficios empresariales; en tanto que la equidad manifiesta la preferencia por destinar recursos para mejorar el bienestar y elevar la calidad de vida de la población (Richardson, 1973).

Si se toma en cuenta de manera particular el proceso de desarrollo regional, se entiende por eficiencia regional la capacidad de las regiones para hacer más efectivo su crecimiento económico, reduciendo el empleo de sus propios recursos. En otras palabras, la eficiencia se refiere a la capacidad de un proceso (económico, social, cultural, ambiental, etcétera) para satisfacer, con sus propios recursos, el crecimiento económico de su población. Por equidad regional se entiende la capacidad de las regiones para reducir al mínimo las desigualdades internas y externas del bienestar de su población. Por otra parte, la sustentabilidad se considera la opción para promover el desarrollo económico y social en equilibrio con la utilización de los recursos naturales que poseen las regiones. Hoy en día se reconoce que si una región no posee niveles de sustentabilidad aceptables, de nada le serviría lograr altos niveles de equidad y eficiencia, pues éstos, tarde o temprano, perderían su sustento, ya que las regiones sustentables son contextos espacio-temporales capaces de asegurar en el corto, mediano y largo plazos el bienestar y la riqueza económica (y de todo tipo) de la población que alberga. El nivel de sustentabilidad varía de un alto potencial de sustentabilidad - supuestamente el nivel ideal porque garantiza la sobreviviencia de las sociedades en el largo plazo— al de bajo potencial — quizás considerado en un nivel crítico, porque sólo garantiza en el corto plazo un ambiente adecuado para la sociedad.

Al ser la región un conjunto complejo de elementos, está sujeta a la entropía, entendida ésta como una medida del desorden derivado de la interacción de los procesos económicos, sociales, culturales, políticos y ecológicos de la misma; y como una medida de la capacidad que ésta posee para satisfacer las necesidades económicas y de bienestar de la población humana. Si una región experimenta un aumento de su entropía, se considera más desordenada porque posee más estados o tendencias de comportamiento posibles. La entropía de la región está ligada a su funcionalidad, a su capacidad de atraer empresas y actividades para localizarse en ella. 
La entropía regional puede clasificarse como entropía absoluta y entropía relati$v a$. La entropía absoluta puede representarse por la relación

$$
\mathrm{S}=-\sum \mathrm{p}_{\mathrm{i}} \mathrm{L}_{\mathrm{N}}\left(\mathrm{p}_{\mathrm{i}}\right)
$$

donde $\mathrm{S}$ : entropía absoluta; $\mathrm{L}_{\mathrm{N}}$ : logaritmo natural; $\mathrm{p}_{\mathrm{i}}$ : probabilidad del evento i; $\mathrm{n}$ : es el número de eventos del aspecto considerado en la región (por ejemplo, respecto con su ecología, economía, etcétera, o, como en el presente artículo, en su relación con el proceso de desarrollo). La entropía regional, de cualquier tipo, es adimensional.

Puede obtenerse el coeficiente de la eficiencia $\left(\mathrm{E}_{\mathrm{Fi}}\right)$ al dividir el valor del indicador del desarrollo entre el valor de la entropía absoluta.

$$
\mathrm{E}_{\mathrm{Fi}}=\mathrm{D}_{\mathrm{i}} / \mathrm{S}_{\mathrm{i}} \quad(2)
$$

Para fines prácticos, conviene determinar en \% el nivel de la eficiencia que puede lograr una región, con la siguiente operación:

$$
\left.\mathrm{E}_{\mathrm{F}}=100-\left[\left(\left(\mathrm{E}_{\text {Fideal }}-\mathrm{E}_{\mathrm{Fi}}\right) / \mathrm{E}_{\mathrm{Fideal}}\right)\right) \times 100\right]=100-\left[\left(1-\mathrm{D}_{\mathrm{i}}\right) \mathrm{x} 100\right] \quad(2 \mathrm{a})
$$

donde $\mathrm{E}_{\mathrm{i}}$ : eficiencia o potencial de entropía en $\%$; $\mathrm{E}_{\mathrm{Fideal}}$ : valor ideal del indicador de $\mathrm{E}_{\mathrm{F}}$; $\mathrm{E}_{\mathrm{Fi}}$ : valor del indicador de $\mathrm{E}_{\mathrm{F}}$ durante el periodo analizado; $\mathrm{D}_{\mathrm{i}}$ : indicador del desarrollo para la región. $\mathrm{E}_{\mathrm{F}}$ proporciona información de la capacidad de las regiones para hacer más efectivo su crecimiento económico y su bienestar, y del mismo también puede deducirse el potencial de desórdenes $\left(\mathrm{P}_{\mathrm{i}}\right.$ en \%) que posee la región por medio de la relación

$$
P_{i}=100-E_{F} \quad(2 b)
$$

Para medir con un solo número que en la región haya eventos terminales con más probabilidades que otros, se utiliza el concepto de entropía relativa. Ésta vale uno si todos los eventos terminales tienen la misma probabilidad, y vale cero cuando sólo hay un evento con la seguridad total de realizarse con respecto a los demás (Arranz, 1993). La entropía relativa $S_{\text {rel }}$ se define como

$$
\mathrm{S}_{\mathrm{rel}}=\mathrm{S} / \mathrm{S}_{\max }=\mathrm{S} / \mathrm{L}_{\mathrm{N}}(\mathrm{n})
$$


Por consiguiente, la entropía relativa es un indicador de la equidad que posee la región: entre más cercano a cero, la iniquidad regional es mayor. Puede obtenerse un indicador de la equidad intrarregional, si se considera el "coeficiente de la equidad" $\left(\mathrm{E}_{\mathrm{Qi}}\right)$ al dividir el valor del indicador del desarrollo entre el valor de la entropía relativa, es decir

$$
\mathrm{E}_{\mathrm{Qi}}=\mathrm{D}_{\mathrm{i}} / \mathrm{S}_{\text {rel }}
$$

cuando $\mathrm{E}_{\mathrm{Qi}}$ menor de 1, la iniquidad es alta; cuando $\mathrm{E}_{\mathrm{Qi}}$ iguala o se acerca a 1, la iniquidad tiende a desaparecer.

\section{Entropía y desarrollo}

La importancia de la entropía es que ésta puede influir en el desarrollo al intervenir en la competitividad y la eficiencia regional. La "competitividad regional" puede entenderse como la capacidad de una región para adelantar a otras regiones en el acceso al conocimiento y tecnología, a los recursos públicos y privados (materiales y financieros), así como al mercado para la venta de sus productos o la adquisición de recursos naturales y materias primas. La región que en un momento consigue adelantar a las demás genera un aumento de su desarrollo, mejora su acceso a los recursos públicos y privados, y asimismo logra una presencia un poco más segura en el mercado. Lo anterior sugiere que si se desea mejorar la competitividad de las regiones, no basta planificar sólo el crecimiento económico o incluso aisladamente el bienestar esperado, sino que también se debe ejercer el control de la entropía regional.

El nivel de la entropía que posee la región puede orientar las acciones con respecto a la planificación de la equidad, la eficiencia y la sustentabilidad del desarrollo regional. Si se toma en cuenta la planificación estratégica, las regiones pueden clasificarse como:

I. Regiones con baja $\mathrm{E}_{\mathrm{F}}$ (eficiencia): son lugares donde las debilidades y amenazas pesan más que sus fortalezas y oportunidades, lo cual obstaculiza su competitividad y el logro de su equidad. En éstas conviene planificar su desarrollo tratando de reducir su dependencia del exterior, y combatir su pobreza y marginación.

II. Regiones con alta $\mathrm{E}_{\mathrm{F}}$ (eficiencia): son zonas en donde sus fortalezas y oportunidades ya superan sus debilidades y amenazas, y permiten impulsar mejor su competitividad. En éstas conviene planificar su desarrollo tratando de aprovechar al máximo la infraestructura (comunicaciones, agua potable, drenaje, alcantarillado, transporte), los recursos naturales de la región, así como las oportunidades que ofrecen los mercados internos y externos. 
III. Regiones con baja $\mathrm{E}_{\mathrm{Q}}$ (equidad): aquí existen desequilibrios intrarregionales notorios. Conviene planificar su desarrollo tratando de reducir su dependencia con el exterior, y combatir su pobreza, marginación y emigración.

IV. Regiones con alta $\mathrm{E}_{\mathrm{Q}}$ : son lugares que han alcanzado un cierto equilibrio intrarregional. En éstos el desarrollo se puede planificar tratando de aumentar las oportunidades y la competitividad que han logrado.

Desde el punto de vista sustentable, a la clasificación anterior habría que agregar la posibilidad que los tipos de regiones sean sustentables en el corto (bajo potencial de sustentabilidad), mediano (mediano potencial de sustentabilidad) o largo plazos (alto potencial de sustentabilidad), lo cual amplía la gama de clasificación de las regiones.

Las regiones con más desventajas son las que combinan los tipos I-III con una sustentabilidad de bajo potencial, pues son lugares con alta dependencia, desigualdades internas, con debilidades (pobreza y marginación) y con un deterioro de su contexto espacio-temporal natural. Las regiones con más ventajas son las que combinan los tipos II-IV con alto potencial de sustentabilidad, pues éstas tienen menos desigualdades internas, dependen más de sus fortalezas y oportunidades endógenas, y presentan opciones de su contexto espacio-temporal-natural que garantiza el desarrollo a largo plazo. Los tipos de región I-IV y II-III con posibilidades de mediano y alto potencial de sustentabilidad se ubican en un punto intermedio.

\section{Procedimiento metodológico}

Los pasos observados para la obtención, el manejo y la interpretación de la información que apoya los argumentos de este artículo se indican a continuación.

1. Aunque en México existen regionalizaciones administrativas y nodales, se prefirió trabajar con una región homogéneo-geográfica, pues este tipo de regionalización se sustenta en aspectos metodológicos rigurosos desde el punto de vista de la ciencia regional y, sobre todo, permite efectuar comparaciones y análisis de los diversos espacios en que puede dividirse el territorio. Con base en este criterio se efectuó la delimitación de las regiones del país (conjuntos de estados) para analizarlas. Éstas son: noroeste (Baja California, Baja California Sur, Sinaloa y Sonora), norte (Coahuila, Chihuahua y Durango), noreste (Nuevo León y Tamaulipas), centronorte (Aguascalientes, San Luis Potosí y Zacatecas), centro-occidente (Colima, Guanajuato, Jalisco, Michoacán y Nayarit), centro, también llamada centro-este (Distrito Federal, México, Hidalgo, Morelos, Puebla, Querétaro y Tlaxcala), sur (Chiapas, Guerrero y Oaxaca), este (Tabasco y Veracruz) y península (Campeche, Quintana Roo y Yucatán). La fuente respectiva es Bassols (1999). 
2. Se obtuvieron los datos de las entidades federativas de México del "Índice de desarrollo humano" (IDH) (PNUD-ONU, 2002 y 2004). También se obtuvieron los datos de las entidades federativas de México de "Migración" (INEGI, 2006), indicadores de los desórdenes sociales como "Porcentajes de presuntos delincuentes registrados en juzgados de primera instancia de los fueros común o federal por homicidio

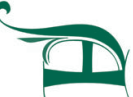
por entidad federativa, 2001"; "Porcentajes de casos comprobados de maltrato infantil presentados ante el Ministerio Público por entidad federativa, 2001"; datos tomados de INEGI, 2002; "Estadísticas Judiciales en Materia penal", edición, 2003, tomo III, pp. 515-516; datos del "Índice de deserción por entidad federativa y sexo según nivel educativo, 2000"; "Tenencia de la vivienda por entidad federativa, 2000"; "Población desocupada por entidad federativa según sexo, 2002", datos tomados de www.inegi.gob.mx, 29 de enero, 2002. Los datos de la flora y fauna por entidad federativa fueron tomados de www.conabio.gob.mx/biodiversidad/ col.htm\#biodiv; 14 de febrero, 2000. El tipo de riesgo natural que presentan las entidades federativas fueron consultados en http://coremi01.coremisgm.gob.mx/ cenapred/temasRiesgos.php, 16 de enero de 2006 del CENAPRED. Los datos anteriores se agruparon para las regiones de México consideradas en el paso 1.

3. Se determinaron las probabilidades de ocurrencia del indicador del desarrollo mediante la obtención de las frecuencias relativas por año, es decir

$$
\mathrm{p}_{\mathrm{i}}=\mathrm{v}_{\mathrm{i}} / \mathrm{V}_{\mathrm{T}} \quad(5)
$$

donde $\mathrm{p}_{\mathrm{i}}$ : probabilidad; $\mathrm{v}_{\mathrm{i}}$ : valor del indicador para el año $\mathrm{i} ; \mathrm{V}_{\mathrm{T}}=\sum \mathrm{v}_{\mathrm{i}}$.

4. Con las probabilidades del punto 3 se obtuvo el valor de la "entropía absoluta" para los indicadores por entidad federativa y región durante el periodo 1950-2003, según la relación indicada por la ecuación (1).

5. Con las probabilidades del punto 3 se obtuvo el valor de la "entropía relativa" para los indicadores por entidad federativa y región durante el periodo 1950-2003, según la relación indicada por la ecuación (3).

6. Con los valores de los pasos 4 y 5 se obtuvieron datos del coeficiente de la "entropía absoluta" $\left(\mathrm{E}_{\mathrm{F}}\right)$ según la ecuación (2), y del coeficiente de la "entropía relativa" $\left(\mathrm{E}_{\mathrm{Q}}\right)$ según la ecuación (4).

7. Los indicadores de los puntos 5 y 6 se agruparon por regiones (paso 1 ) cuando fue necesario.

8. Para determinar el nivel de la eficiencia que puede lograr el país o cada región se aplicó la ecuación 2a, y para determinar el "potencial de entropía" la ecuación $2 \mathrm{~b}$. 
9. Para definir la "distancia euclidiana" de la región hacia la "equidad ideal" se toma como referencia la ecuación 4, así como la relación

$$
\mathrm{e}_{\mathrm{i}}=\mathrm{e}_{\text {Qideal }}-\mathrm{e}_{\mathrm{Qi}}
$$

la cual se estandariza considerando que el valor $\mathrm{e}_{\mathrm{Qi}}$ más cercano a $\mathrm{e}_{\mathrm{Qideal}}$ posee un valor igual a 1. El resto de las distancias estandarizadas se determina por la relación

$$
\mathrm{e}_{\mathrm{i}}=\mathrm{e}_{\mathrm{Qi}} / \mathrm{e}_{\text {Qideal }}(6 \mathrm{a})
$$

10. Los "desequilibrios regionales" (ds) se midieron con la sumatoria de las distancias al desarrollo, la equidad y la eficiencia menos 3 (los parámetros considerados: equidad, eficiencia y sustentabilidad), es decir

$$
\mathrm{ds}_{\mathrm{i}}=\sum\left(\mathrm{e}_{\mathrm{i}}-3\right)
$$

Finalmente, la desigualdad regional $(\delta)$ se mide por la ecuación:

$$
\delta=-\sum\left(\mathrm{ds}_{\min }-\mathrm{ds}_{\mathrm{i}}\right)
$$

donde $\mathrm{ds}_{\min }$ : desequilibrio mínimo de las regiones; $\mathrm{ds}_{\mathrm{i}}$ : desequilibrios de las regiones mayores al desequilibrio mínimo. Si $\delta>0$, entonces existen desigualdades.

11. Para evaluar el potencial de sustentabilidad de las regiones, que debido a la escasez de datos solamente se aplicó para el análisis del año 2003, se elaboró un índice estandarizado para facilitar los cálculos de la entropía de los datos indicados en el punto 2 de la presente metodología.

12. Con este índice estandarizado se procedió a repetir los pasos del 3 al 10 de la presente metodología. Finalmente, con los datos obtenidos de los desequilibrios de la sustentabilidad se elaboró una interpretación del potencial de las regiones según su clasificación en bajo, mediano y alto potencial de sustentabilidad.

\section{Resultados}

\section{Contexto del desarrollo regional en México}

De 1950 a 1982 estuvo vigente el modelo económico de la "sustitución de importaciones", el cual tuvo como motor principal del desarrollo regional la actividad guberna- 
mental. A partir de los años ochenta en México inició el periodo del "neoliberalismo", con el cual el desarrollo ha dependido fundamentalmente de la acción y actividad de los empresarios privados, los organismos internacionales y la banca, así como las industrias transnacionales. Este desarrollo se ha basado en la intención de mejorar la competitividad, la productividad, el ahorro, la inversión y reducir el peso económico

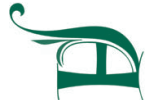
de la estructura gubernamental. A mediados de la década de los noventa la "globalización" adquirió presencia definitiva por medio de la justificación y los esfuerzos por integrar a México al contexto internacional en una posición de competitividad. A partir de estos sucesos, el "desarrollo regional" ha tratado de ser impulsado mediante el crecimiento económico, la competitividad, la integración y participación de las empresas y regiones al proceso exportador, el intercambio y producción de tecnologías de punta, ligadas a las redes productivas y comerciales internacionales. Destacan en este proceso la firma de varios tratados de libre comercio entre México y diversos países (siendo los más notorios los firmados con Estados Unidos y la Unión Europea). Los cambios económicos, demográficos y urbano-regionales del país han provocado que México se caracterice por la concentración de sus actividades económicas en pocos puntos de su territorio. En 1998, por ejemplo, el país tenía 115 ciudades con una población de 50 mil y más habitantes que concentraban $46 \%$ de la población y $95 \%$ del Valor Bruto de la Producción (VBP) industrial, comercial y de servicios del país, es decir, prácticamente toda la riqueza generada en México (Sobrino, 2003:456-459).

Desde la década de los cincuenta el país ha mantenido un aumento constante de su “desarrollo humano" (en el cual el valor de este índice fue 0.48 , que creció a 0.802 en el 2002). En este último año, el Programa de las Naciones Unidas para el Desarrollo ubicó a México en la categoría de desarrollo humano alto. El país ocupaba el quincuagésimo tercer lugar en la clasificación mundial (de un total de 174 naciones). El Distrito Federal y Nuevo León ocupaban las primeras posiciones en tanto que estados como Guerrero, Oaxaca y Chiapas de la región sur se ubicaron en los últimos lugares de la clasificación nacional. Las regiones de mayor desarrollo relativo fueron el noreste (con un índice de desarrollo humano de 0.83 ), norte (0.814), noroeste (0.812), península (0.807) y centro (0.789), seguidas de las regiones centro-norte (0.783), centro-occidente (0.775), este ( 0.757$)$ y sur (0.718). Por lo que respecta al bienestar, para el año 2000 la región sur manifestó la más alta marginación.

\section{La entropía y el desarrollo regional en México}

En el ámbito específicamente regional, durante el periodo 1950-2003 las regiones han evolucionado gradualmente hacia mejores niveles de equidad y eficiencia, pero para 
el año 2003 la mayor eficiencia, por encima del promedio nacional, la habían alcanzado las regiones noreste $(83 \%)$, noroeste $(81 \%)$, norte $(81 \%)$ y la península $(81 \%)$. El resto, centro $(79 \%)$, centro-norte $(78 \%)$, centro-occidente $(77 \%)$, este $(76 \%)$ y sur (72\%), no superaba el promedio nacional de eficiencia (79\%). Respecto a la equidad, las regiones mejor ubicadas por poseer mejores condiciones al promedio nacional (1.24 distancias a la equidad ideal) eran noreste (1.00), norte (1.08), noroeste (1.10), península (1.12) y centro (1.23). Por encima del promedio nacional se encontraban las regiones con mayores niveles de iniquidad: centro-norte (1.26), centro-occidente (1.31), este (1.41) y sur (1.64). En este contexto, y como resumen de los indicadores anteriores, los mayores desequilibrios producto del desarrollo los manifestaban las regiones sur (muy alto) y este (alto), por encima del promedio nacional que poseía un nivel de desequilibrio medio; las regiones centro-norte y centro-occidente presentaban un desequilibrio medio también; en tanto que el resto de las regiones presentaba desequilibrios inferiores a la media nacional: noreste (muy bajo), norte (muy bajo), noroeste (muy bajo) y centro (bajo) (véase cuadro 1).

En este contexto, y consideradas en una perspectiva de largo plazo (1950-2003), la región sur manifiesta las condiciones más desfavorables, en tanto que la noreste presenta las condiciones más favorables. Las regiones que mejor han evolucionado en los indicadores del desarrollo durante este periodo son la noreste, norte, noroeste y la península, en tanto que el centro y el sur son las regiones que han venido perdiendo terreno en ambos aspectos: la primera por mantenerse prácticamente estancada, y la segunda porque sus indicadores evolucionan más lentamente que en el resto de las regiones durante el periodo considerado. El centro-occidente y el este son regiones que han venido evolucionando de manera errática en este proceso. Esta situación tiende a reflejarse en el potencial de desórdenes que manifiestan las regiones, en orden creciente: noreste $(17.19 \%)$, norte $(18.61 \%)$, noroeste $(18.84 \%)$, península $(19.32 \%)$, centro $(21.08 \%)$, centro-norte $(21.66 \%)$, centro-occidente $(22.51 \%)$, este $(24.30 \%)$ y sur $(28.21 \%)$ (véase cuadro 1$)$.

Si se efectúa una comparación entre los periodos 1950-1980 (representativo de la “sustitución de importaciones") y 1980-2003 (representativo del "neoliberalismo"), se observa que en lo que respecta al nivel de desarrollo, éste ha sido superior (0.775) con el neoliberalismo que con la sustitución de importaciones (0.584). Por lo que respecta a la entropía absoluta y relativa, ambas se mantienen constantes durante ambos periodos (con un valor de 2.196 y 0.9996 respectivamente, indicando que las diferencias regionales derivadas de los indicadores del desarrollo han tenido poca variación en ambos periodos). La entropía regional da cuenta que la sustitución de 
importaciones y el neoliberalismo no han resuelto los problemas que plantean la equidad y la eficiencia para asegurar la armonía regional. En términos generales, en ambos casos se ha generado un desarrollo regional con baja equidad y baja eficiencia $\mathrm{y}$, por consiguiente, con desigualdades regionales. A este respecto, se observa que las desigualdades han aumentado de manera no lineal de 1950 (con un valor de 2.633)

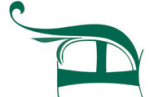
a 2003 (con un valor de 6.462). El menor nivel fue registrado en los años cincuenta (con un valor de 2.633), 4.835 (en la década de los sesenta), 5.789 (en los setenta), 5.076 (en los ochenta), en tanto que el mayor nivel fue alcanzado durante los años noventa (con un valor de 9.614), que ha venido disminuyendo gradualmente (7.190 en 1995, 6.763 en 2000 y 6.462 en 2003). El promedio general de las desigualdades regionales en México ha sido de 6.045, muy superior al manifestado en la década de los cincuenta. Por periodos, en 1980-2003 (durante el neoliberalismo) se han presentado las mayores desigualdades (un promedio de 7.021), comparativamente con el periodo 1950-2003 durante la sustitución de importaciones (con un valor promedio de 4.583) (véase cuadro 2).

Ahora bien, las desigualdades y desequilibrios regionales del país tienden a complicarse si a ellas se aúna el análisis correspondiente a la sustentabilidad. Al respecto, durante 2003 se observó que el promedio del desequilibrio de las regiones ocasionado por el desarrollo fue de 0.718 , en tanto que el promedio de los desequilibrios regionales debido a problemas de sustentabilidad fue de 6.292 (véase cuadro 1), valor que indica que en las regiones las diferencias de sustentabilidad son mayores, y que los principales problemas regionales del país en gran medida provienen de la sustentabilidad de sus regiones en la actualidad. Por regiones, el sur y el este presentan opciones sustentables de alto potencial, por encima de la media nacional, cuya tendencia asegura una sustentabilidad de mediano potencial, al igual que la región noroeste. El resto de las regiones (norte, noreste, centro-norte, centro-occidente, centro y la península) manifiesta posibilidades de sustentabilidad de bajo potencial.

Al retornar a la tipología propuesta en este trabajo, las regiones centro-norte, centro-occidente, sur y este son del tipo I-III, es decir, son regiones con una baja competitividad, desequilibrios internos y escasez de oportunidades económicas. El resto de las regiones es del tipo I-IV (noroeste, norte, noreste, centro, y península), es decir, son regiones con un desarrollo relativamente equitativo pero con una baja competitividad. En términos generales, cuando en el análisis se incluye el factor sustentabilidad, se observa la tendencia de que las regiones con más ventajas en su eficiencia son las que tienden a poseer más desventajas en su sustentabilidad, situación que debe superarse si se desea asegurar una mayor competitividad en las regiones del país. 


\section{Conclusiones}

El análisis precedente confirma la hipótesis de esté artículo de que las desigualdades regionales producto del desarrollo han aumentado en México durante el periodo 1950-2003, y que en fechas recientes los desequilibrios regionales identificados por medio de la evaluación de la sustentabilidad regional aún tienden a ser mayores.

Estas reflexiones sugieren que para alcanzar parámetros de una mayor equidad y una mayor eficiencia sin acelerar los desórdenes regionales, se requiere emprender el desarrollo desde una perspectiva de planificación regional basada en pautas o normas no sólo de cantidad sino de calidad del desarrollo, que permitan proponer metas y objetivos, y regular los procesos, así como ordenar los procedimientos para el manejo de los recursos requeridos. El potencial de desórdenes que aún poseen las regiones de México hace necesario que el futuro desarrollo regional que se emprenda esté basado en una planificación estratégica sustentable.

¿Qué propician las "desigualdades regionales" en México en la actualidad? El presente artículo no puede responder plenamente a esta interrogante, pues preferentemente da cuenta de la descripción de su evolución y situación actual. Pero al respecto pueden adelantarse algunas conjeturas relacionadas con el desarrollo sustentable:

1. Las políticas públicas se han inclinado a la eficiencia por sobre la equidad y la sustentabilidad, y el "eficientismo" generado ha exigido la concentración de los recursos públicos en las regiones con mayores ventajas económicas, lo que ha dado pie a un círculo vicioso, que genera preferencias hacia las "regiones más competitivas", y discriminación de las "regiones más pobres" al distribuir el presupuesto público en el ámbito territorial.

2. La equidad ha sido subordinada al crecimiento económico y a la competitividad. Las políticas públicas en México han ignorado que el bienestar social requiere un manejo intencionado, autónomo y humanista: no necesariamente es la consecuencia lógica de una "eficiencia económica de corte empresarial".

3. La "sustentabilidad" ha sido una variable cuya ponderación sólo ha adquirido relevancia en años recientes, sobre todo debido a la preocupación por el agotamiento de los recursos naturales estratégicos como el petróleo, y porque el cambio climático está ocasionando estragos en el ámbito territorial: tampoco es considerada una variable que requiere reglas y recursos propios, y que debe manejarse con autonomía de los intereses políticos particulares, pues es una prioridad nacional. En resumen, las desigualdades territoriales en México son producto de la búsqueda de una calidad de vida cada vez más compleja, en cuya base se encuentra el manejo de las políticas públicas, y por consiguiente, son resultado de decisiones 
sociales y políticas discriminatorias hacia el equilibrio territorial, pero que a su vez están realimentando su propio círculo vicioso en el ámbito espacial: el del aumento de los desórdenes territoriales como la emigración, la pobreza, la marginación, y aun la posible fractura territorial de la nación debido a la iniquidad y a la pérdida de sustentabilidad de sus regiones.

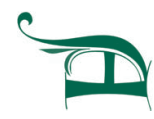

\section{Bibliografía}

Appendini Kirsten, Daniel Murayama, Rosa Ma. Domínguez, "Desarrollo Desigual en México, 1900 y 1960", en Demografía y Economía, México, vol. vi, núm. 1, 1972.

Arranz Ramones, Antonio, Planificación y control de proyectos, México, Grupo Noriega Editores, 1993.

Bassols Batalla, Ángel, "Investigaciones urbanas y regionales de México: ¿para conocer o transformar una realidad?", en Humberto Muñoz (coord.), La sociedad mexicana frente al tercer milenio, México, Miguel Ángel Porrúa-Coordinación de Humanidades, 1999.

Boisier, Sergio, Modernidad y Territorio, Santiago de Chile, Instituto Latinoamericano y del Caribe de Planeación Económica y Social, CEPAL, 1996.

Garza, Gustavo, "Tendencias de las desigualdades urbanas y regionales en México 1970-1996", en Estudios Demográficos y Urbanos. México, num.3, vol. 15, COLMEX, 1997.

Hernández Laos, Enrique, "La desigualdad regional en México", en Rolando Cordera Campos y Carlos Tello (coords.) en $L a$ desigualdad en México, México, Siglo XXI, 1984.
INEGI, Sistema de Cuentas Nacionales de México, www.inegi.gob.mx, 29 de enero de 2002.

, Población inmigrante, emigrante y saldo neto migratorio por entidad federativa 2000, www.inegi.gob.mx, 14 de enero de 2006.

PNUD-ONU, Informe sobre Desarrollo Humano México 2002, México, 2002 y 2004.

Ramírez, María Delfina, "Las desigualdades interregionales en México de 1970 a 1980", en Estudios Demográficos y Urbanos. México, vol. 1, núm. 33, COLMEX, 1986.

Ruíz, Chiapetto Cresencio, "Desigualdades Regionales en México 1900 a 1993”, en Estudios Demográficos y Urbanos, México, vol. 11, núm. 3, COLMEX, 1997.

Richardson, Harry W., Economía Regional, Barcelona, Vicens Vives, 1973.

Shannon, C. E., "Mathematical Theory of Communication", Bell System Technical Journal, vol. 27, 1948.

Sobrino, Jaime, Competitividad de las ciudades en México, México, COLMEX, 2003.

Theil, H., Economics and information Theory, Chicago, Rand Mc Nally and Company, 1967. 
Anexo

Cuadro 1

México: Eficiencia, equidad, desequilibrios, sustentabilidad y potencial de desórdenes de sus regiones, 2003

\begin{tabular}{lcclcllc}
\hline Región & $\begin{array}{c}\text { Nivel de } \\
\text { eficiencia (\%) }\end{array}$ & $\begin{array}{c}\text { Distancia a } \\
\text { la equidad } \\
\text { ideal }\end{array}$ & $\begin{array}{c}\text { Nivel de } \\
\text { desequilibrio } \\
\text { del desarrollo }\end{array}$ & $\begin{array}{c}\text { Desequilibrios } \\
\text { sustentabilidad }\end{array}$ & $\begin{array}{c}\text { Tipo de } \\
\text { región }\end{array}$ & $\begin{array}{c}\text { Con susten- } \\
\text { tabilidad de }\end{array}$ & $\begin{array}{c}\text { Potencial } \\
\text { de desórdenes }\end{array}$ \\
\hline Noroeste & 81 & 1.10 & 0.288 Muy bajo & 6.038 & I-IV & Mediano potencial & 18.84 \\
Norte & 81 & 1.08 & 0.247 Muy bajo & 9.070 & I-IV & Bajo potencial & 18.61 \\
Noreste & 83 & 1.00 & 0.000 Muy bajo & 8.170 & I-IV & Bajo potencial & 17.19 \\
Centro-Norte & 78 & 1.26 & 0.780 Medio & 10.247 & I-III & Bajo potencial & 21.66 \\
Centro-Occidente & 77 & 1.31 & 0.929 Medio & 6.578 & I-III & Bajo potencial & 22.51 \\
Centro & 79 & 1.23 & 0.679 Bajo & 7.096 & I-IV & Bajo potencial & 21.08 \\
Sur & 72 & 1.64 & 1.925 Muy alto & 0.000 & I-III & Alto potencial & 28.21 \\
Este & 76 & 1.41 & 1.241 Alto & 2.098 & I-III & Alto potencial & 24.30 \\
Península & 81 & 1.12 & 0.371 Muy bajo & 7.328 & I-IV & Bajo potencial & 19.32 \\
\hline Promedio & 79 & 1.24 & 0.718 Medio & 6.292 & I-III & Mediano potencial & 21.30 \\
\hline
\end{tabular}

Fuente: Elaborado con datos del "Índice de Desarrollo Humano" (PNUD-ONU, 2002 y 2004) y el "Procedimiento metodológico" de este artículo.

Cuadro 2

México: Desigualdades regionales de su desarrollo 1950-2003

\begin{tabular}{cccccccccc}
\hline Región & 1950 & 1960 & 1970 & 1980 & 1990 & 1995 & 2000 & 2003 & Promedio \\
\hline Desigualdad & 2.633 & 4.835 & 5.789 & 5.076 & 9.614 & 7.190 & 6.763 & 6.462 & 6.045 \\
\hline
\end{tabular}

Fuente: Elaborado con datos del "Índice de Desarrollo Humano" (PNUD-ONU 2002 y 2004), y el "Procedimiento metodológico" de este artículo. 